\title{
L'aménagement hydroélectrique de Souapiti sur le fleuve Konkouré, en Guinée
}

\author{
The Souapiti hydro-electric development \\ on the Konkouré, Guinea
}

\author{
PAR P. SIMON \\ INGÉNIEUR EN CHEF A BLECTRICITÉ DE FHANCE \\ INSPECTION GÉNÉRALE POUR I'UNRON FRANGASE ET G'ÉTRANGER
}

\begin{abstract}
Suscilée par les perspectives d'implantation en Guinée d'une industrie de l'aluminium à partir de gisements de balnate importants, l'étude des possibilités d'aménagement du Konkouré, dirigée par E.D.F. a partir de 1952, a conduit a prévoir deux usines hydro-silectriques: Sonapiti et Amaria.

A Solapiti, in barrage de $120 \mathrm{~m}$ de hautenr. constitué en matérian latéritique compacti $\left(25.10^{6} \mathrm{~m}^{*}\right)$, créera une retenue de 11 milliards de in ${ }^{3}$, suffisante pour assurer ane complete régnlarisation interannuelle des débits. Un évacuaten de crues et une vidange de fond seront raccordes chacun à l'une des deux dérivalions provisoires. Une centrale souterraine, située sous l'appui rive droite du barrage, sert Equipe de 4 groupes vertican de $133 \mathrm{MVA}$. Enfin, une galerie de fuite de $114 \mathrm{~m}^{2}$ de section restituera les eatrx turbinces à $6 \mathrm{~km}$ environ de l'nsine. La puissance de la centrale atteindra $500 \mathrm{MW}$ et sa productibilité annnelle. $32 \mathrm{mil}$ liards de liWh.
\end{abstract}

Le Konkouré est connu depuis très longtemps! Le comte de Sanderval, qui fut sans doute le premier explorateur à remonter le fleuve, il y a plus d'un demi-siècle, a rapporté une documentation précieuse à l'époque, en particulier des fameuses chutes de Kaleta.

Plus récemment, en 1944, une mission Péchiney de prospection des gisements de bauxite s'est intéressée au Konkouré. Cette mission a reconnu que le Konkouré était le seul fleuve en Guinée susceptible de fournir les quantités d'énergie nécessaire à une usine d'aluminium.

Quatre ans plus tard, en 1948-1949, une mission d'Electricité de France mit en évidence les possibilités réelles du Konkouré et proposa un schéma d'équipement complet du fleuve.

Dès lors, la vallée du Konkouré apparut comme une heureuse conjonction entre l'énorme potenliel d'énergie électrique et les gisements de

\begin{abstract}
Proposals to establish an aluminium industry to exploit extensive bauxite deposits, caused the F.I.F. to start investigating the possibility of developing the konliowe in 1952. The outcome of this investigation was to recommend the building of two hydro-electric power stations: Souapiti and Amaria.

$1120 \mathrm{~m}$ high dam made of compacted laterites $\left(25 \times 10^{5} \mathrm{~m}^{\prime \prime}\right)$ will be used al Souapifi to form a $11,000,000,000 \mathrm{~m}^{3}$ reservoir which will be capable of ensuring completely regular flow from one year to the next. A spillway and a hottom sluice will be connected to each of the two temporary diversion tunnels. An underground power house, located ander the right abutment, will be equipped with four $133 \mathrm{MVA}$ bertical units. A tailrace tunnel with a $114 \mathrm{~m}^{*}$ tross section will return water to the river some $3 \mathrm{~km}$ dounslream of the polver house. The power developed by the power house will be as much as $500 \mathrm{MW}$ and the annual oulput $3,200,000,000 \mathrm{kWh}$.
\end{abstract}

bauxite trouvée dans cette vallée, le tout situé à proximité -.- une centaine de kilomètres — de l'excellent port de Conakry.

Le Konkouré a un bassin versant de 17000 $\mathrm{km}^{2}$ et une Iongueur de $360 \mathrm{~km}$.

Les précipitations atmosphériques, qui dépassent $4 \mathrm{~m}$ et parfois $5 \mathrm{~m}$ sur la côte, atteignent encore une moyenne de $2,50 \mathrm{~m}$ sur l'ensemble du bassin. Malheureusement, l'alternance régulière des saisons sèches et des saisons de pluies a pour grave conséquence une très grande irrégularité des cours d'eau. Pour le Konkouré, les débits varient de $10 \mathrm{~m}^{3} / \mathrm{s}$ à l'étiage à 2 à 3000 $\mathrm{m}^{3} / \mathrm{s}$ en hautes eaux.

Le premier problème à résoudre pour l'équipement du Konkouré était done celui de la régularisation du régime hydraulique. Problème dont on mesure toute l'envergure lorsqu'on sait que $50 \%$ du volume d'eau annuel du fleuve 
s'écoule pendant deux mois, et $80 \%$ pendant quatre mois.

L'étude précise de l'aménagement du Konkouré, confiée en 1951 à l'Electricité de France - Inspection Générale pour l'Union Française et l'Etranger -_.. et à l'Energie Electrique de Guinée, a abouti à un schéma d'aménagement susceptible de produire plus de 5 milliards de
kWh régularisés et cela dans des conditions particulièrement économiques.

Cette production sera le fait de deux usines, celle de Souapiti, dont les études sont entièrement terminées et qui fournirait à elle seule 3 milliards 200 millions de $\mathrm{kWh}$, et d'une deuxième usine - Amaria - située à une quarantaine de km a l'aval.

\section{CONCEPTION DU PROJET DE SOUAPITI}

Le Konkouré présente une succession de biel's, de rapides et de chutes. Les chutes les plus importantes sont celles de Kaleta avec une dénivellée de $40 \mathrm{~m}$.

A l'amont de ces chutes, le Konkouré coule dans une plaine largement ouverte, pour se resserrer au site de Souapiti.

Il était donc tentant d'établir un barrage à cet endroit, situé à $7 \mathrm{~km}$ en amont des chutes.

L'étude économique du barrage a conduit à adopter un ouvage de $120 \mathrm{~m}$ de hauteur, permettant d'accumuler 11 milliards de $\mathrm{m}^{3}$, dont 6 sont destinés à la régularisation annuelle et interannuelle des débits.

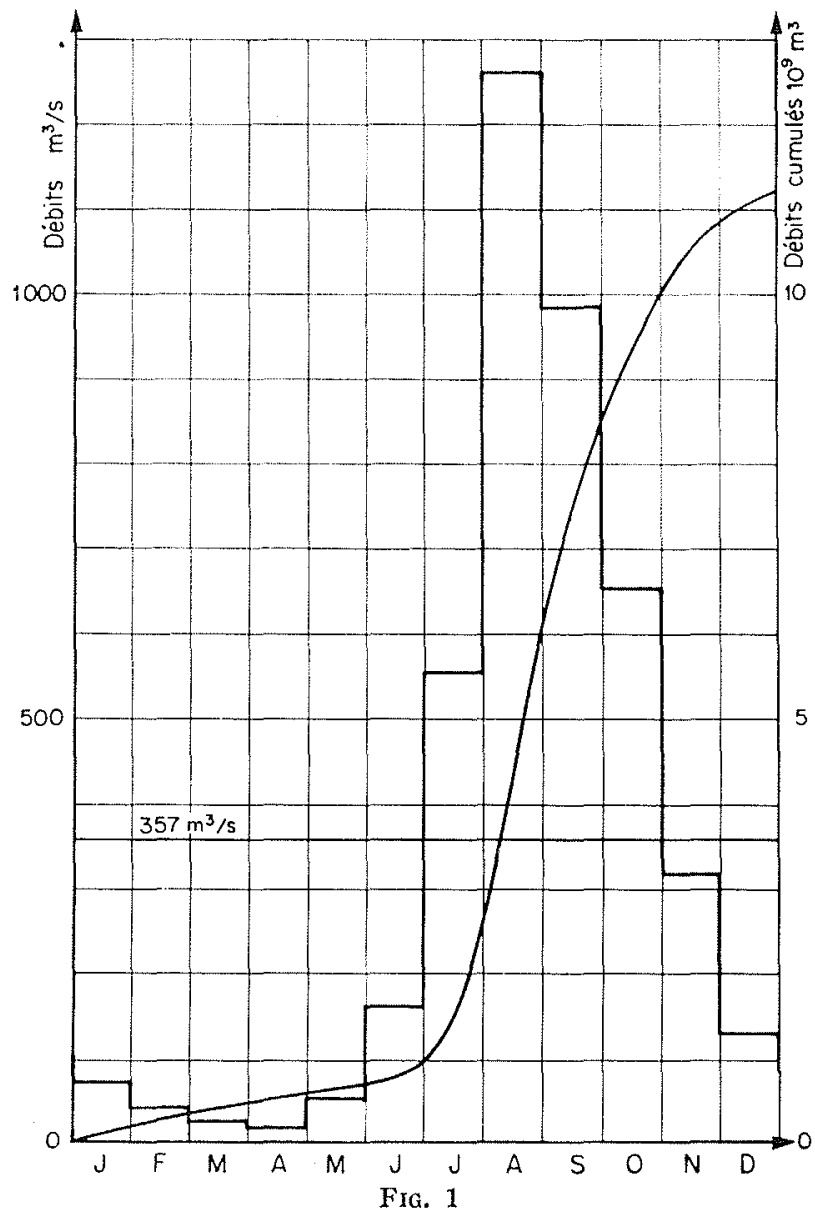

La hauteur de chute maximum utilisce sera de $165 \mathrm{~m}$, compte tenu de la hauteur du barrage, des chutes de Kaleta et de la pente du fleuve.

Pour cela, on a été amené à prévoir une centrale en souterrain et une galerie de fuile de $6 \mathrm{~km}$ de longueur.

Ainsi concu, l'aménagement de Souapiti permet de satisfaire aux conditions imposées par l'industrie de l'aluminium, c'est-à-dire de fournir l'énergie sous une puissance, constante en tous temps, de $370000 \mathrm{~kW}$.

\section{Travaux d'études.}

Depuis 1952, les campagnes d'études sur le Konkouré et à Souapili se sont succédé sans interruption; elles ont porté notamment sur :

l'hydrologie,

la topographie,

la géologie et les recomnaissances des terres.

\section{Hydrologie.}

Le bassin du Konkouré est soumis au régime ropical de transition, caractérisé par l'alternance d'une saison seche avec une saison humide d'égale importance.

L'étude hydrologique est basée d'une part sur les mesures directes de débit effectuées pendant onze ans, d'autre part sur trente années d'observations pluviométriques intéressant l'ensemble du bassin.

On a ainsi pu établir avec une tres bonne approximation le module du Konkouré, ainsi que les variations mensuelles de débils et les écarts entre les années extrêmes.

La hauteur d'eau moyenne tombant sur le bassin versant à Souapiti est de $2050 \mathrm{~mm}$. La hauteur d'eau écoulée est de $1040 \mathrm{~mm}$, correspondant à un module interannuel de $357 \mathrm{~m}^{3} / \mathrm{s}$, c'est-à-dire à un apport annuel de 11 milliards de $\mathrm{m}^{3}$.

En année sèche, le volume d'apport peut des- 


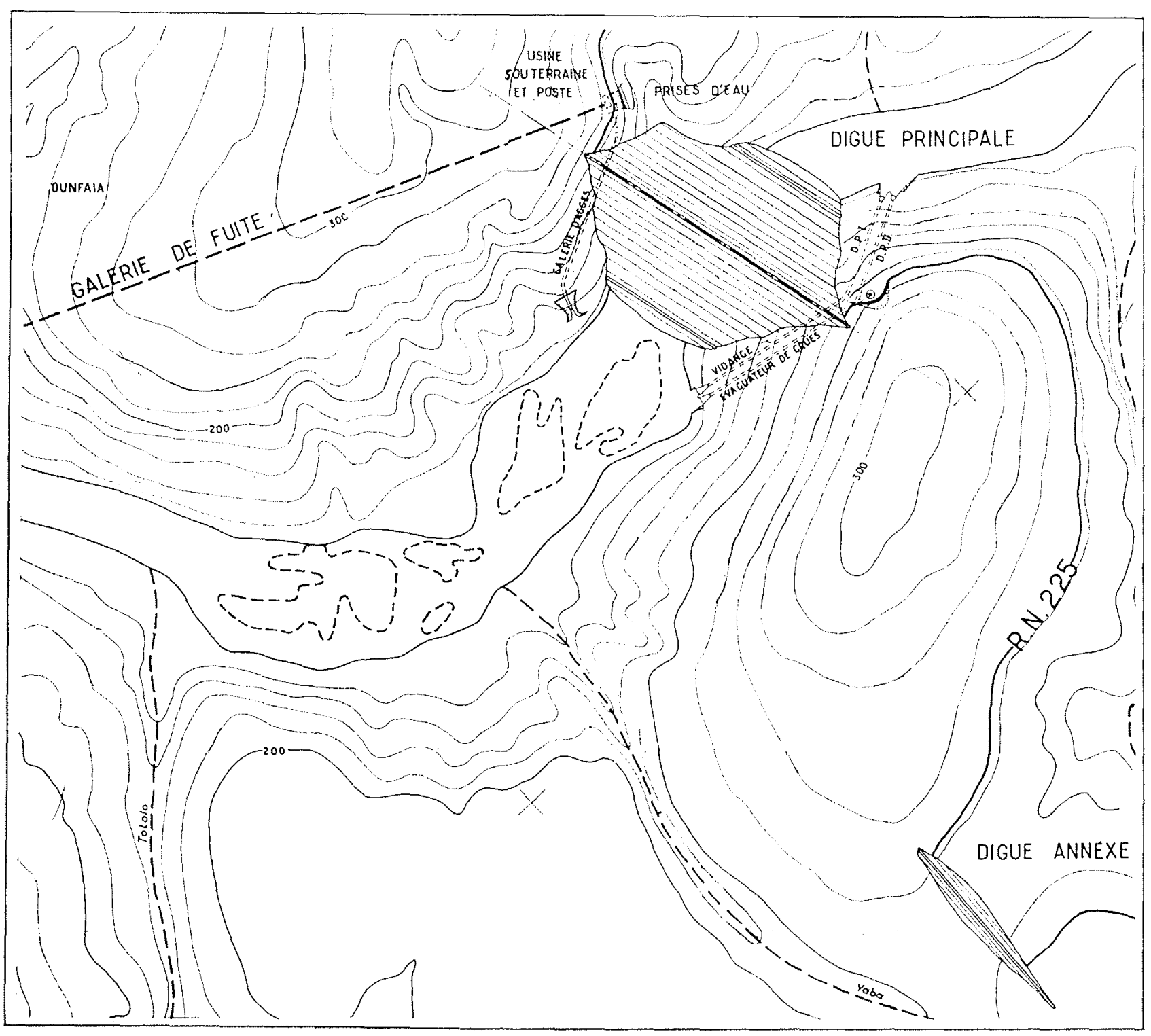

FIG. 2

cendre à 8 milliards, alors qu'il atteint 16 milliards en année très humide.

Les écarts de débits au cours de l'année sont considérables : $10 \mathrm{~mm}^{3} / \mathrm{s}$ environ à l'étiage et $3000 \mathrm{~m}: \mathrm{s}$ pour la plus forte crue connue, qui a d'ailleurs été mesurée par jaugeages.

Une étude statistique des pluies provoquant les plus fortes crues a permis d'évaluer la crue millénaire à $4000 \mathrm{~m}^{3} / \mathrm{s}$. Cependant cette étude vise essentiellement à prévoir le volume d'eau d'une crue de plusieurs semaines, en raison du volume de laminage considérable dont on dispose dans la retenue de Souapiti (2,5 milliards).

L'humidité de l'air est importante: l'hygro. métrie atteint et dépasse même $95 \%$ le matin en tontes saisons et $50 \%$ environ an milieu de la journée; elle peut descendre parfois à $20 \%$ en saison sèche.

La température demeure toujours élevée. Les mois les plus chauds sont mars et avril avec des températures maxima moyennes de $40^{\circ} \mathrm{C}$ et des pointes atteignant $42^{\circ}$, mais, pendant la nuit, la température descend de 22 à $20^{\circ} \mathrm{C}$ environ. Au cour's de la saison des pluies, la température est souvent moins élevée de $10^{\circ}$, aussi bien de jour que de nuit, par rapport aux tempélatures extrêmes de la saison sèche.

Des essais d'évaporation ont été faits pendant plusieurs années a l'aide de cuves diversement situées. Ils conduisent à admettre une éraporation de $1,50 \mathrm{~m}$ sur un plan d'eau de srande ctenduc. Pendant la saison sèche, l'eva- 
poration crô̂t en mème temps que la température pour atteindre $7,4 \mathrm{~mm}$ par jour en mars; pendant la saison des pluies, l'humidité de l'air ne descendant pas en dessous de $55 \%$, la moyenne journalicre n'est que de $2,8 \mathrm{~mm}$.

La perte par évapotranspiration dans la zone de la retenue s'élève à $1100 \mathrm{~mm}$, de sorte que la perte annuelle nette due à la retenue sera de $400 \mathrm{~mm}$ par an, c'est-à-dire de l'ordre de 150 millions de $\mathrm{m}^{3}$, chiffre très faible $(1,3 \%)$ visi-vis du rolume d'eau emmagasiné.

\section{Travaux topographiques.}

Les études du Konkouré commencèrent par la photographie aérienne de la région intéressée et l'établissement des cartes au $1 / 50000^{\circ}$, la seule carte existant auparavant étant à l'échelle du $1 / 200000^{\circ}$.

D'autre part, des équipes de topographes ont relevé tout d'abord tous les emplacements possibles de barrages, travail qui a servi de base a l'élude générale de l'aménagement du Konkouré; puis ces équipes ont procédé aux levés à grande échelle des emplacements des ouvrages.

\section{Géologie.}

L'étude géologique de Souapiti a porté :

1) sur l'étanchéité de la cuvette de retenue,

2) sur la structure superficielle et profonde de la zone destinée à supporter ou à recevoir les ouvrages,

3) sur la reconnaissance des zones d'emprunt de terres pour la construction de la digue.

Le projet de Souapiti est essentiellement intéressé par la formation du primaire, constitué ici par des niveaux horizontaux de grès et de schistes.

A travers cet ensemble, sont venues des roches éruptives basiques dont le type est une dolérite. Cette lave, montant sous forme de cheminées, s'est répandue au sein des schistes, où elle constitue des sills, c'est-à-dire des filons concordants.

En pratique, nous avons affaire, à l'amont, dans la zone de la cuvette, à des grès, et plus à l'aval, dans la zone des ouvrages, à une alternance de bancs sensiblement horizontaux de schistes et de dolérites.

Toutes ces roches sont recouvertes d'un épais manteau d'altération.

Des phénomènes chimiques et physiques en rapport avec le climat (où alternent régulière. ment saison sèche et saison des pluies) ont amené une altération rapide des roches avec éclatement des silicates. C'est le phénomène dit de latérisation, qui a provoqué la formation de terres rouges, relativement meubles en profondeur, avec concentration vers le haut des oxydes de fer et d'alumine sous forme de carapaces dures.

La cuvette de retenue de Souapiti a lait l'objet de nombreuses prospections (a pied, par avion, par hélicoptère) et d'une étude photogéoJogique. Les observations ont permis de conclure à l'étanchéité de la retenue.

Sur le site des ouvrages, de nombreux sondages en profondeur, représentant une longueur totale de $2000 \mathrm{~m}$, ont été effectués. Des puisatiers indigènes, suivant les méthodes des chercheurs d'or de la Haute-Guinée, ont creusé dans les terrains de couverture plus de 200 puits de reconnaissance arec des profondeurs dépassant parfois $20 \mathrm{~m}$. Ces reconnaissances ont d'ailleurs été complétées par des sondages à la sondeuse hélicö̈dale el surtout par le creusement de grandes tranchées donnant ainsi une coupe des terrains jusqu'à $20 \mathrm{~m}$ de profondeur en certains points.

Pour compléter l'étude géologiciue, un puits de reconnaissance a été descendu sur $103 \mathrm{~m}$ de profondeur dans l'axe d'une conduite forcée. Une Galerie de courte distance excavée depuis le fond lu puits a permis de se rendre compte de la honne tenue de la roche en place, la dolérite, au niveau de la vô̂te de l'usine.

\section{Travaux sur les terres.}

Pour obtenir toute garantic sur les possibilités d'ériger une digue de grande hauteur en terre latéritique, de nombreux essais et mesures ont été faits, aussi bien en laboratoire que sur le terrain même.

Les essais en laboratoire ont porté sur les terrains de fondations du barrage et sur les terres d'emprunt devant servir de matériaux constitutifs de l'ouvrage.

Ces essais et mesures, au nombre de plus d'un millier, ont été effectués :

A Paris : au laboratoire « Mécasol » et au laboratoire du Bâtiment et des Travaux $\mathrm{Pu}-$ blics,

A Gap : au laboratoire de Mécanique des Sols d'E.D.F.,

Sur le site de Souapiti : au Iaboratoire du chantier.

Des essais ont également été faits par le laboratoire de Mécanique des Sols de Vicksburg aux Etats-Unis, à la demande de M. Turnbull, Ingénieur-conseil américain, spécialiste des grands barrages en terre.

Un essai d'excavation des terres en carrière et des essais de compactage en vraie grandeur ont été exécutés sur le site de Souapiti avec un rouleau compacteur à pneus de 50 tonnes et un rouleau à pieds de mouton.

Ces essais ont fait l'objet d'un rapport présenté en septembre 1958 au Sixième Congrès International des Grands Barrages. 


\section{DISPOSITIONS ET DESCRIPTION DES OUVRAGES}

\section{La digue principale.}

Cet ouvage a été étudié par le Bureau d'études A. Coyne et J. Bellier, qui nous a également prêté son concours pour l'exécution des essais de compactage.

La digue s'élève à $121 \mathrm{~m}$ au-dessus du lit du lleuve. Sa longueur en crête est de $1075 \mathrm{~m}$ el sa largeur de $10 \mathrm{~m}$. Son volume est de 25 millions de $\mathrm{m}^{3}$. Les pentes, variables, sont en moyenne de $3 / 1$, d'où une largeur, suivant le lit du fleure, de $700 \mathrm{~m}$ environ.

La digue est constituée par un massif homogène imperméable de terres compactées, flanqué a l'aval d'un massif semi-perméable ou perméable de matériaux tout venant.

Les deux massifs sont séparés par un filtre incliné.

Les talus sont protégés par une couche d'enrochement.

Les matériaux utilisés sont des terres siltoargileuses provenant des terrains de couverture situés immédiatement à l'amont et à l'aval de l'emplacement du barrage.

Le massif semi-perméable ou perméable est constitué par la partie supérieure des zones d'emprunt qui est constitué lui-même d'une croûte ou carapace plus on moins dure de latérite.

Les enrochements proviennent egalement de cette carapace dure.

En somme, le barrage est constitué de façon a pouvoir utiliser la totalité des matériaux extraits.

Les terres sont mises en place par couches horizontales et compactées au rouleau.
Les essais de compactage ont montré que l'épaisseur des couches devra itre de 35 à $45 \mathrm{~cm}$ avec le rouleau à pneus de 50 tonnes et le nombre de passages aller et retour du roulean, de 4 .

L'épaisseur des couches compactées au rouleatu à pied de mouton n'est que de $15 \mathrm{~cm}$; le nombre de passages est de 8 allers simples.

Le rouleau à pheus s'est done averé plus efficace que le rouleau à pied de mouton.

Lat teneur en eau optinum est comprise entre 18 et $25 \%$ et la densité sèche correspondante est de 1,55 à 1,75 .

lendant les Iravaux, le chantier sera protégé par un batardeau amont de $30 \mathrm{~m}$ de hauteur incorporé ultérieurement dans le corps de la digue. Il en est de même du batardeau aval.

\section{La digue annexe.}

Une digue annexe de $22 \mathrm{~m}$ de hauteur et $820 \mathrm{~m}$ de longueur sert à fermer un col faisant communiquer la vallée principale avec le thalweg d'un petit affluent.

Cette digue est concue exactement suivant les mèmes principes que la digue principale.

\section{La dérivation provisoire.}

Les eaux du Konkouré sont dérivées dans deux galeries de dérivation provisoire:

- La D.P.I., longue de $720 \mathrm{~m}$, comprend 2 tronçons revêtus, de $10 \mathrm{~m}$ de diamètre, et 1 tronçon non revêtu, de $13,70 \mathrm{~m}$ de diamètre, dont la longueur est de $500 \mathrm{~m}$;

-. La D.P.II, longue de $770 \mathrm{~m}$, comprend 2 tronçons revêtus, de $10 \mathrm{~m}$ de diamètre, et 1 tron-

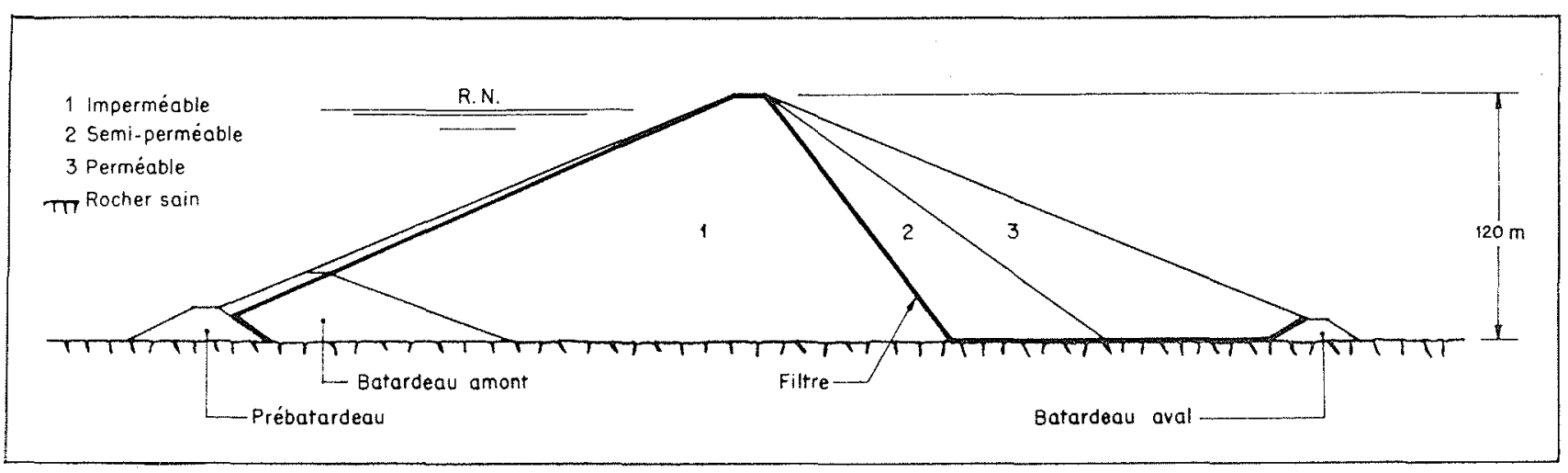

Fri. :3. - Digue principale 
con non revêtu, de $13,70 \mathrm{~m}$ de diamètre, d'une longueur de $250 \mathrm{~m}$.

\section{Ouvrages évacuateurs de crues}

Il est prévu deux ouvrages :

- Un évacuateur de crues de surface, circulaire, raccordé par un puits vertical à l'une des dérivations provisoires,

- Une vidange de demi-fond, raccordée par une galerie plongeante à l'autre dérivation provisoire.

Afin de n'avoir pas à donner des dimensions exagérées à ces ouvrages, on a préféré laminer les crues du Konkouré en emmagasinant une partie de leur volume dans Ia retenue. Une tranche d'eau de $6 \mathrm{~m}$, au-dessus de la cote de retenue normale, est réservée à cet effet derrière le barrage, représentant une capacité de 2 milliards $1 / 2$ de $\mathrm{m}^{3}$.

De plus, un col naturel, situé sur la rive droite du Konkouré à $3 \mathrm{~m}$ en dessous de la retenue de surélévation exceptionnelle, constitue un déversoir naturel de sécurité.

Compte tenu du volume de laminage, il a été possible de dimensiomer l'évacuateur de crue pour $2250 \mathrm{~m} / \mathrm{s}$ au maximum, et la vidange de demi-fond pour $500 \mathrm{~m}^{3} / \mathrm{s}$.

L'évacuateur de crue a un diamètre de $32.10 \mathrm{~m}$.

Son raccordement à l'une des dérivations provisoires - la D.P.I. - se fait par puits vertical et galerie inclinée de $10 \mathrm{~m}$ de diamètre et de $140 \mathrm{~m}$ de longueur totale. Le fonctionnement de cette dérivation provisoire est prévu à écoulement libre.

La vidange de demi-fond est destinée à fonctionner dès que le plan d'eau derrière le barrage dépassera le niveau de retenue normal. Elle est équipée par une vanne de garde de $3,50 \times 6,50 \mathrm{~m}$ et une vanne de manœuvre de $3,25 \times 6 \mathrm{~m}$ sous $75 \mathrm{~m}$ de charge. La galerie plongeante de raccordement à la dérivation provisoire (la D.P. II) a $6 \mathrm{~m}$ de diamètre et $110 \mathrm{~m}$ de longueur. La galerie de dérivation fonctionnera alors en charge. Pour éviter une vitesse d'écoulement trop grande dans la partie non revêtue, des redents ont été disposés à l'entrée de la galerie et un étranglement a été aménagé à son extrémité aval. De plus, un reniflard assure l'évacuation de l'air vers la galerie de l'évacuateur de crue fonctionnant à écoulement libre.

Ces dispositions ont été mises au point par des essais sur modèle réduit.

\section{Les prises d'eau et conduites d'amenées.}

Quatre prises d'eau identiques, situées sur la rive droite, alimentent chacune une turbine par une conduite d'amenée verticale de $4,80 \mathrm{~m}$ de diamètre et de $120 \mathrm{~m}$ de longueur.

La fermeture est assurée par 4 vannes de garde de $3,50 \times 5,75 \mathrm{~m}$ sous $56 \mathrm{~m}$ de charge.

Le batardage est prévu par une vanne unique de $3,50 \times 7,40 \mathrm{~m}$ pouvant ètre déplacée d'une prise à l'autre.

Les quatre conduites d'amenée sont plus exactement des puits excavés dans le rocher, revêtus et chemisés.

\section{La centrale souterraine.}

La centrale souterraine, creusée dans la dolérite sur la rive droite du Konkouré, exige un déroctage de l'ordre de $100000 \mathrm{~m}^{3}$.

Les résultats de sondage ont conduit à ne prévoir aucun revêtement, à l'exception de la voûte supérieure qui sera bétonnée.

La salle des machines a une longueur de $118 \mathrm{~m}$ pour une largeur de $16,50 \mathrm{~m}$.

Une disposition originale permettra d'incorporer les poutres supports des chemins de roule-

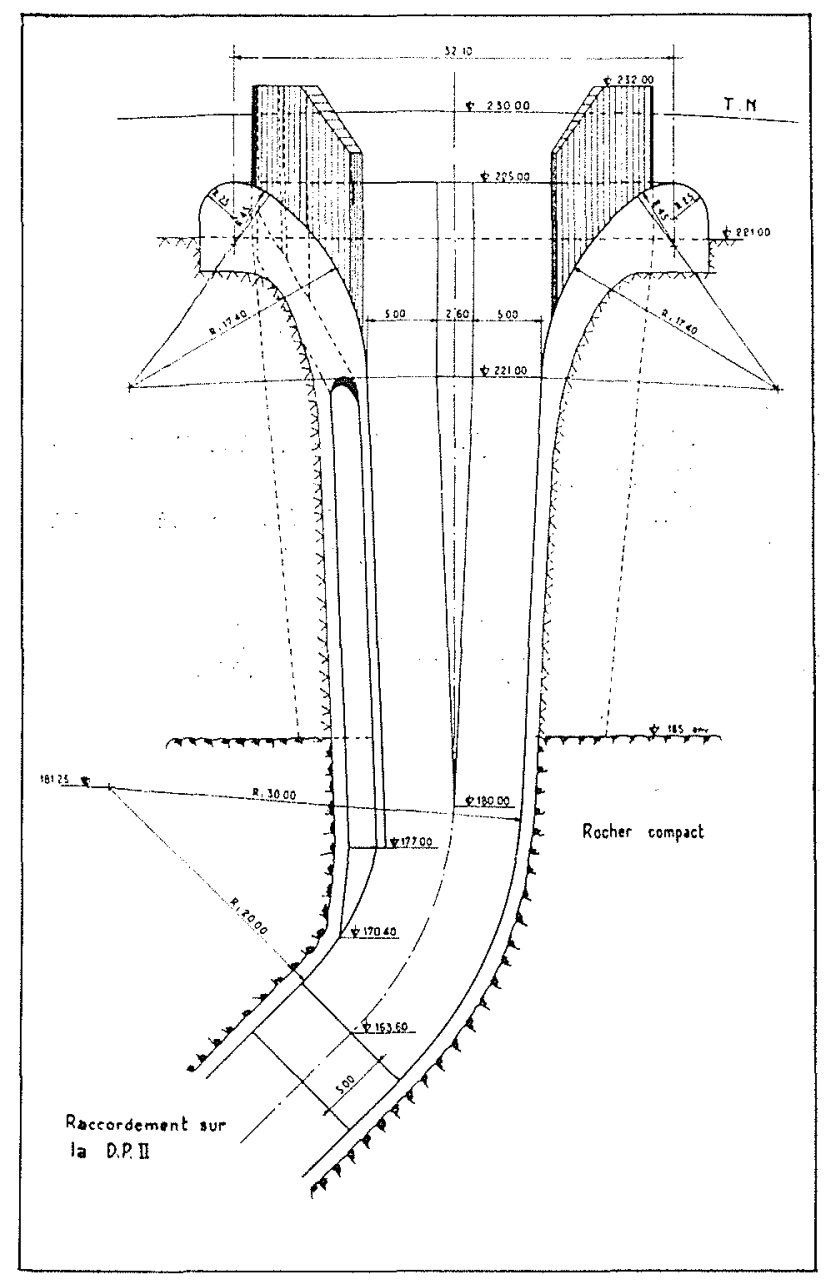

Fic. 4. .-.. Evacuatcur de arues 
ment des ponts roulants aux retombées de la voute. On se dispensera ainsi de construire les poteaux que l'on rencontre habituellement dans les centrales.

De plus, les deux ponts roulants jumelés, de 200 tonnes chacun, seront d'un type spécial, de facon à réduire au maximum la hauteur de la centrale.

La centrale renferme quatre groupes Francis verticaux, dont l'un demeure en réserve.

Cette réserve assure le maintien de la puissance constante, en cas d'incident sur un groupe.
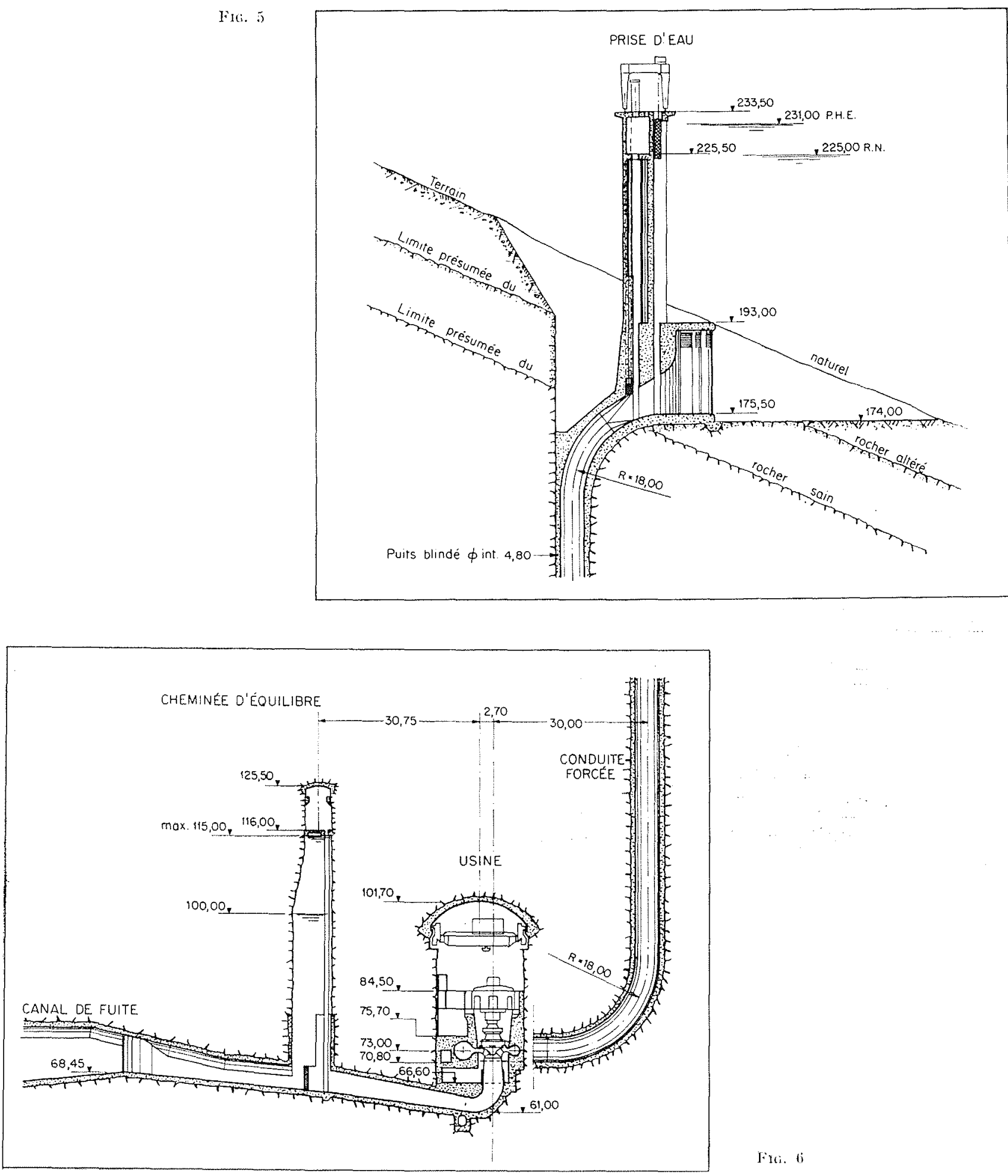
Les turbines, dont la vitesse normale est de $187,5 \mathrm{~m} / \mathrm{mm}$, ont une puissance de $171000 \mathrm{ch}$.

Les alternateurs ont une puissance apparente de 133 MVA sous $\varphi=0,925$.

L'entraxe des groupes est de $17 \mathrm{~m}$. Il est conditionné principalement par la dimension des turbines.

Cette cireonstance a permis de ménager, entre les alternateurs, des losses dans lesquelles il a été possible de loger les quatre transformateurs. Cette dispoistion olrrant par ailleurs toute sécurité -...- les fosses sont entièrement fermées -..., est évidemment très avantageuse.
Deux accès à la centrale ont été prévus :

-... Un accès rapide, par puits vertical, dans lequel circule un ascenseur et qui sert également au passage des câbles $220 \mathrm{kV}$;

- Une galerie de $600 \mathrm{~m}$ de longueur permettant aux véhicules l'accès jusqu'à la salle des machines.

Le poste de départ est situé sur une plateforme reliée à la centrale par un puits d'accès.

Entre la centrale et la galerie de fuite, s'intercale une cheminée d'équilibre dont le rôle n'apparaîtrait qu'en deuxième étape, après con-

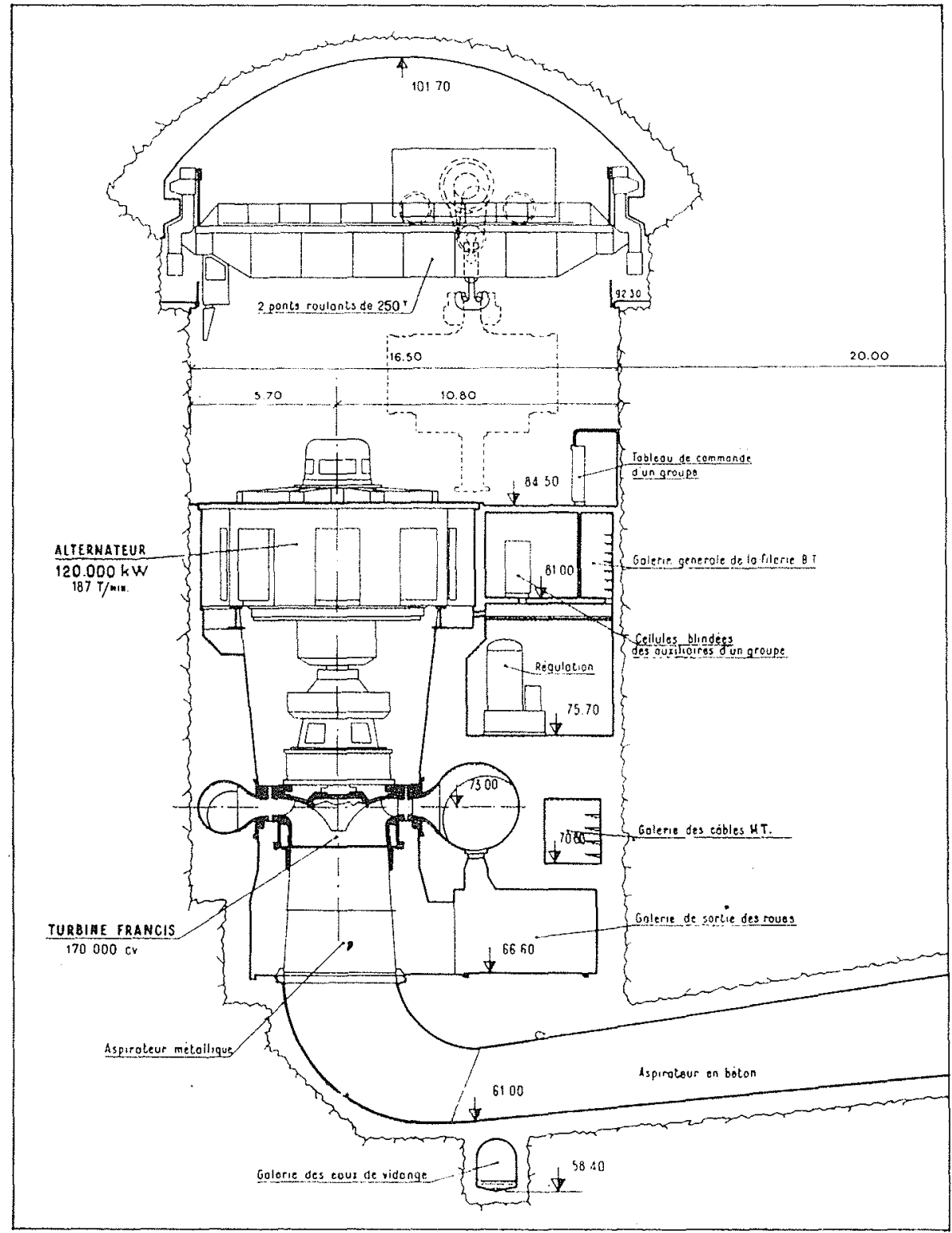

Fro. 7. -... Usine Coupe dans laxe d'un groupe 


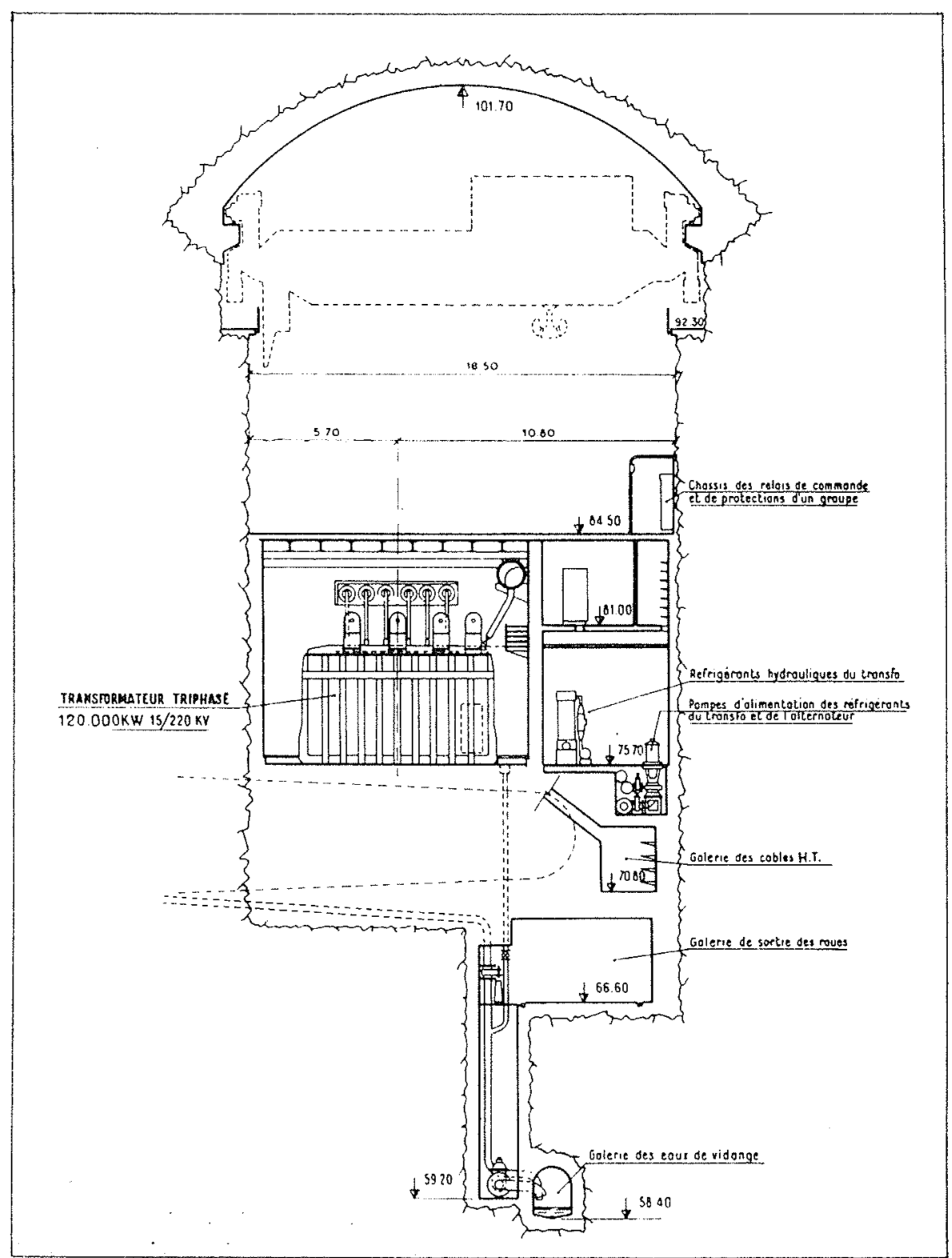

Fig. 8. - Usine. Coupe entre deux groupes

struction du barrage d'Amaria. Ce barrage mettrait en charge la galerie de fuite de Souapiti.

\section{La galerie de fuite.}

La restitution, dans le Konkouré, des débits turbinés, s'opère par une galerie de fuite, de $114 \mathrm{~m}^{2}$ de section, inscrite dans un carré de $11 \mathrm{~m}$ de côté avec une voûte en calotte de $8,60 \mathrm{~m}$ de rayon. Elle a $6200 \mathrm{~m}$ de longueur et débouche à l'aval des chutes de Kaleta. La bonne qualité du rocher a permis de ne pas prévoir en principe le revètement de cette galeric.

Elle est calculée pour un débit de $340 \mathrm{~m} \% \mathrm{~s}$.
Sa pente est de $1,95 \%$ sur $4800 \mathrm{~m}$ et de $0,50 \%$ dans la partie aval, sur les 1400 derniers mètres. Cette pente est donc descendante de l'usine vers le Konkouré, ce qui facilite les travaux (évacuation des déblais et éventuellement des venues d'eau). Ce choix permet le fonctionnement à écoulement libre é de limiter alors $\dot{a}$ une faible longueur, au voisinage de l'extrémité aval de la galerie, la mise en vitesse de l'eau.

\section{Ouvrage récupérateur d'énergie.}

Le calage du radier de la galerie de fuite a son extrémité aval a été imposé par les ủon- 


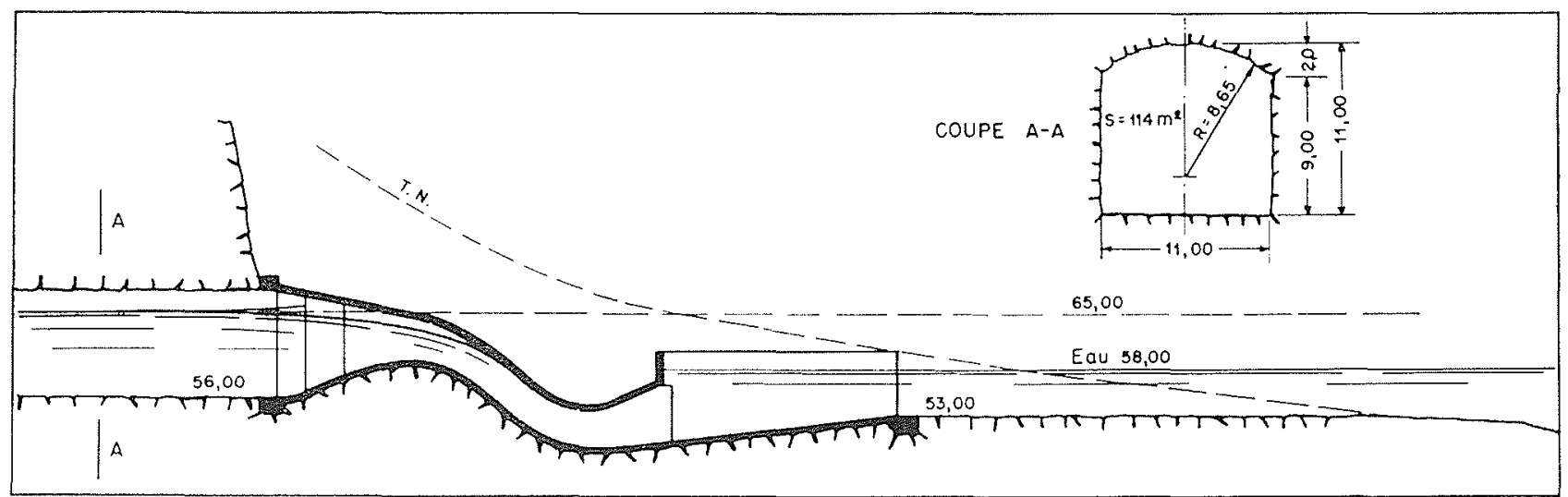

Fig. 9. -...- Réupérateur d'éneryio

nées topographiques de la zone de restitution (cote 56), alors que le niveau des eaux dans le lit du Konkouré est de $2 \mathrm{~m}$ plus haut (cote 58) pour le débit de fonctionnement de l'usine.

Mais en raison de la grande hauteur de la galerie, soit $11 \mathrm{~m}$, imposée par l'importance de ce débit, le niveau aval (cote 65) au débouché de la galerie, est à $7 \mathrm{~m}$ au-dessus du niveau du Konkouré.

Il en résultait que toute la chute disponible n'était pas intégralement utilisée.

La solution consistant à abaisser la galerie et à lui donner une contrepente à l'aval pour récupérer ces quelques mètres de chute, a été écartée pour des raisons d'exécution.

Nous avons alors eu l'idée de prolonger la galerie par un ouvrage de raccordement extérieur, débouchant sous le niveau du Konkouré, a la facon d'un siphon; cette disposition devait permetre l'écoulement "en dépression », que la galerie fonctionne en charge ou à écoulement libre.

Les essais sur modèle (à l'échelle du 1/74), dont fut chargée la SOGREAH, ont effectivement confirmé qu'il y avait récupération de chute.

En fonctionnement à écoulement libre, la dépression s'établit tout le long de la galerie par effet d'entrainement de l'air par l'eau à travers l'ouvrage de raccordement. Ce mode de fonctionnement a été jugé préférable au fonctionnement en charge qui risquait de provoquer une instabilité par suite du décollement du plan d'eau en voùte, en un point quelconque de la galerie.

La dépression réalisée constitue la hauteur do chute récupérée.
Les essais ont ainsi permis d'affirmer que le gain de chute, en toutes circonstances, sera d'au moins $4,50 \mathrm{~m}$, correspondant à un gain d'énergie de 100 millions de kWh.

\section{Pont provisoire. - Durée des travaux.}

L'accès normal au barrage nécessitera la construction d'un troncon de route nouvelle. Pour accéder aux deux rives du Konkouré, sans avoir à attendre la mise en service de cette route, un pont métallique a été jeté sur le fleuve immédiatement à l'aval de l'emplacement du barrage.

Ce pont, construit à partir de la rive gauche sur une longueur de $225 \mathrm{~m}$, est prolongé jusqu'à la berge opposée par une digue en terre compactée de $220 \mathrm{~m}$ de longueur et de $8 \mathrm{~m}$ de hauteur. Ce pont est raccordé à la piste d'accès provisoire au site de Souapiti.

La durée des travaux de l'aménagement de Souapiti est conditionnée d'abord par le temps nécessaire à l'exécution des dérivations provisoires pour pouvoir procéder à la coupure du Konkouré, ensuite par les délais de mise en place des 25 millions de $m^{3}$ des terres de la digue principale.

On peut estimer que les travaux s'étaleront en tout sur six années.

Les travaux de la digue subiront chaque année un arrêt pendant la saison des pluies. On peut compter sur une durée de travail d'environ. 150 jours par saison sèche. Par contre, pour les travaux souterrains, le chantier peut conserver une activité normale toute l'année. 


\section{CONCLUSION}

L'aménagement de Souapiti esl remarquable a divers points de vue:

Le barrage se distingue particulièrement par l'emploi de terres latéritiques qui n'ont dommé lieu jusqu'à présent, dans le monde, qu'à des réalisations rares et modestes; il conslitue igalement par sa taille (hauteur et volume) un des plus grands ouvrages projetés.
Les dimensions de la centrate souteraine of des galeries constituent également un caractère intéressant de l'aménagement.

Enfin la récupération de $4,50 \mathrm{~m}$ de chule, srace à la conception de louvage aval de la galerie de fuite, confere a la restitution de la centrale une originalité qui méritait d'être menlionnée.

\section{I S C U S S I O N}

\section{rrésident : M. Ligovyat}

Après avoir remercié les services delectricite de France et tous ceux qui lui ont apporté leur concours, notamment le Bureau Coyne et J. Bellier, pour la recherche de la solution technigue la plus appropriée au but à atteindre, M. Gunew souligne les difficultés du jroblème posé aux projecteurs et la qualité de leurs travaux.

Il s'agissait de s'accommoder d'un site oì les débits varient de $10 \mathrm{~m}^{3} / \mathrm{s}$ a $2500 \mathrm{~m}^{3} / \mathrm{s}$, el d'une vallée relativement ouverte, pour la création d'une puissance constante, ì niveau suffisamment élevé pour justifier l'installation en Guinée d'une industrie de l'aluminium et a un prix suffisamment bas pour permettre une fabricar tion concurrentielle sur le plan international.

Les projeteurs ont tiré le meilleur parti possible des possibilités du Jonkouré, en recourant notamment it un barrage en terre et à des dispositions judicieuses. Il n'était pas en leur pouvoir d'éviter un prix de liwh relativement élevé et nettement supéricur à celui du Trouilou. La différence est toutefois atténuée par la présence simultanée en Guinéc de bauxite et d'énergice.

M. Alldrrer pose une question sur un detail technique du projet :

"La mise en dépression de l'air de la galerie de fuite ne peut entrâner un gain de puissance que dans la mesuré où les rentrées d'air atmosphérique par défaut d'étanchéité de la galerie n'atteindront qu'un débit faible par rapport au debit d'eau de la centrale. "

«Etant dome la grande longueur de cette galerie de fuite et compte tenu de ce qu'une roche est considérablement plus perméable à l'air qu'a l'eau, cette condition sera-t-elle remplie? 》

N. Smon répond que cette remarque est tout a fait exacte et que, si, dans le sein de la montagne, la roche se présente, d'après les puits de recomaissance, comme extrêmement compacte et sans fissures, par contre, quelques rentrées d'air sont à craindre dans la zone qui avoisine la sortie de la galerie et nécessiteront sans doute quelques colmatages.

M. WIL, pense que, s'il y avait une rentrée d'air, celle-ei proviendrait de lacration des turbines.

M. Smon indique, en réponse à la remarque de M. Wrim, que les turbines sont en communication avec latr atmosphérique par les cheminées d'équilibre et ajoute que, de toute façon, la vitesse de l'eau dans l'ouvrage aval permet d'évacuer une importante quantité d'air; il est d'ailleur's préru, en tète de la galerje, une admission contrôlée de l'air pour permettre de limiter la dépression.

M. Vanter remarque que, si, en général, les débits des cours d'eau de l'Aîrique Equatoriale surclassent nettement ceux des bassins européens, la répartition saisonnière et la valeur spécifique de ees débits ne sont pas très différents de ceux de nos torrents de montagnes : $80 \%$ des apports annuels passent en deux mois dans les cour's d'eau d'Afrique Equatoriale, tandis que les apports de nos torrents atteignent 75 a $80 \%$ de
Gabondance amuelle pendant les trois mois de fusion des neiges; ces maxima se produisent, d'ailleurs, à peu pres atux mèmes saisons (mai-juiin) tandis que l'on obscrve, de part et d'autre, des etiages prolongés en automne ct en hiver. Enfin, le volume accumulable, rapporté a la superficie du bassin versant, est sensiblement le même : 1 million de m" d'eau par lim".

M. le Président souligne que les memes coefficients portent, toutefois, en Afrique Equatoriale, sur des quanlités et des suríaces énormes.

M. Gunser conftrme l'observation du président, en précisant que, pour arriver au résuliat demandé au projeteur, il fallait une réserve considérable que l'on n'aurait jamais pu réaliser en France.

M. Chaportheir, qui a eru apercevoir sur le dessin du barrage de Souapiti un tapis d'étanchécté, demande si lo probleme du contoumement éventuel du barrage par an dessous et par les flanes ne s'est pas posé ì Souapiti comme il s'est posé à Serre-Ponçon qui, selon M. Srmon, présente des analogies avec Souapiti.

M. Ie p'résident indique à M. Chapouthaer que e'est un filtre qu'il a vu, et non un tapis d'étanchéité.

M. Snox confirme que l'étanchéité dans le lit du línkouré est assurée par lat roche en place à l'emplacement du barrage; eette roche est une altemance de dolérite et de schistes tres similaires les uns aux autres et les nombreux sondages faits, tant dans l'axe de la riviere, alu pied du barrage, que pour l'usine et les autres ouvrages, ont montré aux projeteurs que, pratiquement, il n'y avait aucune fuite $\mathrm{ni}$ ancun contoumement a critindre.

M. Srmos précise que les marmites qui ont pu se produire seront purgies et remplies de béton et que le filtre a été ramené à l'aval du barrage pour récolter les eaux. Sur les flanes, les terrains de couverture seront laissés en place. Un parafouille assez important et bourré de terre argileuse est prévu jusqu'au rocher dans les parties basses et moins profondement dans les parties hautes, où la charge est faible.

En réponse à une question de M. Carraier, M. Srmon indique que l'aménagement de Souapiti ne pose pas de probleme important de débil solide, eu ègard à la longueur de la retenue $(60 \mathrm{~km})$ : il n'y a guère que quelques très faibles atterrissements de sable fin provenant, en hautes caux, des bassins supérieurs.

M. Ronier croit nécessaire d'ajouter quelques chiffres it ce que vient de dire M. Smon sur les transports solides. L'Onston et l'E.D.F. ont effectué des mestres do transports solides sur les fleuves du régime tropical do transition : la première sur le Chari et le Logone, la seconde sur la Benoue. Pour les suspensions, on trouve en crue des turbidités de 100 à $200 \mathrm{~g}$ par $\mathrm{m}^{3}$; il s'agit de sable très fin et surtout de vases et de limons, dont une partie notable passera par les ouvages de prise. Pour le charriage, les mesures faites sur la Benoué, pour un bassin de $100000 \mathrm{~km}^{2}$ environ, ont donné 1000000 de m" par an.

M. Je Président remercie M. Ronmen. 